\title{
Quantitative analysis of repaired rabbit supraspinatus tendons ( \pm channeling) using magnetic resonance imaging at 7 Tesla
}

\author{
Guy Trudel $^{1,2,3}$, Samuel Duchesne-Bélanger, ${ }^{1,4}$,Justin Thomas ${ }^{1,4}$, Gerd Melkus ${ }^{5,6}$, Greg O. Cron ${ }^{5,6}$, \\ Peder E. Z. Larson ${ }^{7}$, Mark Schweitzer ${ }^{8}$, Adnan Sheikh ${ }^{5,6}$, Hakim Louati $^{1}$, Odette Laneuville ${ }^{1,4}$ \\ ${ }^{1}$ Bone and Joint Research Laboratory, Ottawa Hospital Research Institute, Ottawa, ON, Canada; ${ }^{2}$ Department of Medicine, Division of Physiatry, \\ University of Ottawa, Ottawa, ON, Canada; ${ }^{3}$ Department of Biochemistry, Microbiology and Immunology, University of Ottawa, Ottawa, ON, \\ Canada; ${ }^{4}$ Department of Biology, University of Ottawa, Ottawa, ON, Canada; ${ }^{5}$ Department of Medical Imaging, The Ottawa Hospital, Ottawa, ON, \\ Canada; ${ }^{6}$ Department of Radiology, University of Ottawa, Ottawa, ON, Canada; ${ }^{7}$ Department of Radiology and Biomedical Imaging, University of \\ California at San Francisco, San Francisco, CA, USA; ${ }^{8}$ Department of Radiology, Stony Brook University NY, New York, NY, USA
}

Contributions: (I) Conception and design: G Trudel, PEZ Larson, M Schweitzer, O Laneuville; (II) Administrative support: G Trudel, O Laneuville; (III) Provision of study materials or patients: G Trudel, O Laneuville; (IV) Collection and assembly of data: G Trudel, S Duchesne-Bélanger, J Thomas, G Melkus, GO Cron, A Sheikh, O Laneuville; (V) Data analysis and interpretation: G Trudel, S Duchesne-Bélanger, G Melkus, GO Cron, A Sheikh, O Laneuville; (VI) Manuscript writing: All authors; (VII) Final approval of manuscript: All authors.

Correspondence to: Dr. Odette Laneuville. Department of Biology, Faculty of Science, Gendron Hall Room 3-372, University of Ottawa, 30 Marie Curie Ottawa, ON K1N 6N5, USA. Email: olaneuvi@uottawa.ca.

Background: The quantitative assessment of supraspinatus tendons by conventional magnetic resonance is limited by low contrast-to-noise ratio (CNR). Magnetic resonance imaging (MRI) scanners operating at 7 Tesla offer high signal-to noise ratio (SNR), low CNR and high spatial resolution that are well-suited for rapidly relaxing tissues like tendons. Few studies have applied T2 and T2* mapping to musculoskeletal imaging and to the rotator cuff tendons. Our objective was to analyze the $\mathrm{T} 2$ and $\mathrm{T} 2$ * relaxation times from surgically repaired supraspinatus tendons and the effect of bone channeling.

Methods: One supraspinatus tendon of 112 adult female New Zealand white rabbits was surgically detached and repaired one week later. Rabbits were randomly assigned to channeling ( $n=64)$ or control $(n=48)$ groups and harvested at 0, 1, 2, and 4 weeks. A 7T magnet was used for signal acquisition. For T2 mapping, a sagittal multi slice 2D multi-echo spin-echo (MESE) CPMG sequence with fat saturation was applied and T2* mapping was performed using a 3D UTE sequence. Magnetic resonance images from supraspinatus tendons were analyzed by two raters. Three regions of interest were manually drawn on the first T2-weighted dataset. For T2 and T2*, different ROI masks were generated to obtain relaxation times.

Results: T2-weighted maps but not $\mathrm{T} 2{ }^{*}$-weighted maps generated reliable signals for relaxation time measurement. Torn supraspinatus tendons had lower T2 than controls at the time of repair $(20.0 \pm 3.4$ vs. 25.6 $\pm 3.9 \mathrm{~ms} ; \mathrm{P}<0.05)$. T2 increased at 1,2 and 4 postoperative weeks: $22.7 \pm 3.1,23.3 \pm 3.9$ and $24.0 \pm 5.1 \mathrm{~ms}$, respectively, and values were significantly different from contralateral supraspinatus tendons $(24.8 \pm 3.1 ; 26.8 \pm 4.3$ and $26.5 \pm 3.6 \mathrm{~ms}$; all $\mathrm{P}<0.05)$. Bone channeling did not affect $\mathrm{T} 2(\mathrm{P}>0.05)$.

Conclusions: Supraspinatus tendons detached for 1 week had shorter T2 relaxation time compared to contralateral as measured with 7T MRI.

Keywords: T2 relaxation times; 7 Tesla; tendon; surgical re-attachment

Submitted Dec 09, 2020. Accepted for publication Mar 26, 2021.

doi: $10.21037 /$ qims-20-1343

View this article at: http://dx.doi.org/10.21037/qims-20-1343 


\section{Introduction}

Magnetic resonance imaging (MRI) plays an important role in the evaluation and prognosis of rotator cuff injuries $(1,2)$. Sher $e t a l$. found the prevalence of supraspinatus (SSP) tendon tears by MRI to be 34\% (3). The large number of asymptomatic tears likely underestimates the true prevalence of rotator cuff tears (2). MRI informs on all of the rotator cuff individual tendons tear sizes, degeneration, inflammation, muscle atrophy and fat accumulation; all crucial information for surgical decision making $(2,4)$. Surgical repair of the SSP tendon is one of the most commonly performed orthopedic surgery, often for highgrade tears $(5,6)$. Functional and anatomical success depend on a number of parameters; larger tears, older patients, fat accumulation in the SSP muscle, and tendons with retraction and degeneration have worse outcomes (7-10). Preoperative MRI was correlated with surgical outcomes at 1 year after full thickness rotator cuff tear (11). As such, MRI provides crucial perioperative diagnostic and prognostic information.

Tendons have a highly ordered structure due to type I collagen assembly into fibrils aggregating to form larger fiber bundle units aligning along the long axis of the tendon (2). Degenerated rotator cuff tendons show altered structure and biochemical composition at the enthesis: higher water content, decreased collagen content, collagen fiber disruption, and altered glycosaminoglycan accumulation (12-15). The effect of a tear on the SSP tendon proper, proximal to the enthesis have not been the object of scrutiny. However, SSP tendon proper is central to the success of a surgical repair since they will hold the sutures and be part of the mechanical chain of force transmission. One study identified the tendon proper as the most common mode of re-rupture, ahead of dehiscence at the repaired enthesis site $(16,17)$.

The quantitative assessment of SSP tendons by conventional MR is limited by low contrast-to-noise ratio (CNR) $(1,2)$. Assessment of tendon structure by MRI at $3 \mathrm{~T}$ is therefore not possible using long echo times (18). At high field MRI using pulse sequences, normal tendons have a uniform low signal intensity with conventional pulse sequences $(2,19)$. MRI scanners operating at 7 Tesla offer higher signal-to noise ratio (SNR) (20), and increased spatial resolution that are well-suited for the study of rapidly relaxing tissues like tendons (21). Specifically, the transverse relaxation time (T2) and the apparent transverse relaxation time $\left(\mathrm{T}_{2}{ }^{*}\right)$ are sensitive to the biochemical content of the tendon proper (19). Both, the T2 and T2*, are influenced by free water. In tissues like tendons, T2 and $\mathrm{T}^{*}$ reflect the organization of the matrix of collagen fibers and hydration level (19). While T2 is a time constant for the decay of the transverse magnetization arising from natural interactions at the atomic or molecular levels, $\mathrm{T}^{*}$ is additionally affected by inhomogeneity in the main magnetic field, and local susceptibility changes. Few studies have applied T2 and T2* mapping to musculoskeletal imaging and to the rotator cuff tendons $(18,22)$. Anz et al. reported SSP tendon T2 mapping in 30 asymptomatic volunteers using $3 \mathrm{~T}$ and described an increasing distal to proximal gradient unaffected by age (21). In another study, T2 mapping -but not $\mathrm{T} 2{ }^{*}$ of 8 volunteers on a $3 \mathrm{~T}$ scanner positively correlated SSP tendon tear size (23). Short T2* were suggested as reliable indicators for tendinopathies (22). Ultrashort echo time (UTE) and variable echo time sequences have been designed to acquire increased signal from tendons; 7T scanners using UTE allowed for robust mono- and bi-exponential $\mathrm{T}^{*}$ mapping of tendons (22). 7T-MR scanners have quantitatively assessed the structure and biochemical properties of some tendons (e.g., Achilles) $(24,25)$. However, to our knowledge, no quantitative $7 \mathrm{~T}$ MR normative values of SSP tendons are available.

Various augmentation procedures have been investigated to improve the success rate of SSP repair. One of them is bone channeling. Creating holes in the humeral head at the SSP insertion footprint could promote mesenchymal stem cell migration from the red marrow. Twenty-fold increase in cellularity was measured 1-2 weeks after channeling in the animal model (26). However, channeling did not improve enthesis reformation after SSP repair in the rabbit model (27). The effect of channeling on the SSP tendon proper remains unknown.

The purpose of our study was to generate $\mathrm{T} 2$ and $\mathrm{T} 2$ * maps of rabbit supraspinatus tendons proper and show the effects of a SSP tendon tear, of surgical repair up to 4 postoperative weeks and of bone channeling compared to the contralateral SSP tendon. Our hypotheses were that (I) detached SSP tendons will show signs of disuse; (II) surgical SSP repair will restore tendon T2 and T2* times incrementally with postoperative duration and (III) channeling will not influence SSP tendon repair.

\section{Methods}

\section{Animal model}

Animal procedures were approved by the Institutional 


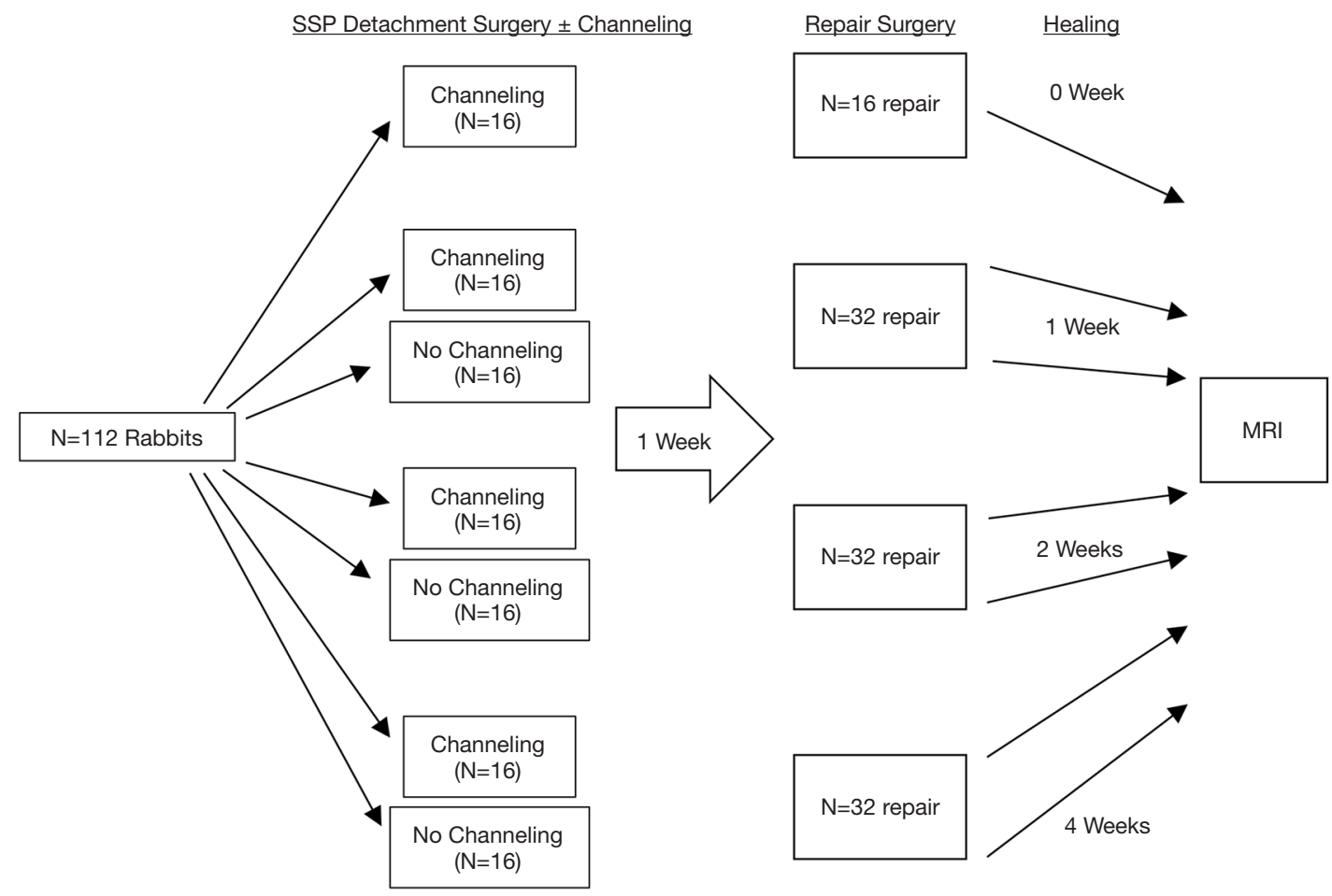

Figure 1 Experimental design. The total number of rabbits and numbers in individual groups are indicated.

Animal Care Committee under protocol \#ME-184. One hundred and twelve female New Zealand white rabbits $(3 \mathrm{~kg})$ were housed individually at $22^{\circ} \mathrm{C}$ on a 12 -h light/dark cycle with access to water and standard rabbit chow (Charles River Saint-Constant, Quebec, Canada). The experimental design and materials were used in a previous investigation where the supraspinatus enthesis reformation of rabbits after surgical repair was quantified by MR at $7 \mathrm{~T}$ using $\mathrm{T} 2$ mapping (27). The current investigation reports findings on the repaired SSP tendon and contralateral tendons.

\section{Surgical detachment and tendon repair}

Each rabbit underwent two surgeries to one randomly selected shoulder: an initial SSP tendon detachment at the humeral head insertion was performed to simulate a complete tendon tear followed by a repair surgery one week later (28). The experimental design and sample size for individual groups is illustrated in Figure 1. Bone channeling at the humeral head SSP footprint was carried out at the detachment surgery in 64 rabbits while the remaining $48 \mathrm{did}$ not receive the intervention. Channeling consisted of drilling four 1-mm-diameter and 10-mm-depth holes to ensure communication with the subchondral bone marrow. After detachment, the tendon was wrapped in a polyvinylidene membrane (5 $\mu \mathrm{m}$, Durapore; Millipore Corp., Bedford, MA, USA) to promote nutrition diffusion and prevent tendon adhesion to surrounding tissue in both the channeling and no channeling rabbit groups. After one week, the incision was re-opened, and a curette was used to clear the insertion site. The Millipore membrane was removed and the retracted SSP tendon was pulled laterally and attached to a $3 \mathrm{~mm}$ Bio-FASTak $^{\circledR}$ anchor (Arthrex Inc. Naples, FL, USA) using an inverted horizontal mattress \#2 FiberWire suture (29). Rabbits were sacrificed at $0(\mathrm{n}=16), 1 \quad(\mathrm{n}=32), 2(\mathrm{n}=32)$, or $4(\mathrm{n}=32)$ postoperative weeks and both shoulders were dissected en bloc with precaution to harvest the complete SSP muscle and to preserve its new attachment site to the humeral head. Shoulders were wrapped in saline gauze and frozen at $-40^{\circ} \mathrm{C}$ until imaged.

\section{Image acquisition}

Prior to MRI, the shoulders were thawed for $12 \mathrm{~h}$ at $4{ }^{\circ} \mathrm{C}$. A $7 \mathrm{~T}$ (31 cm bore size) superconducting magnet (Agilent/GE MR 901 system) equipped with actively shielded imaging 
gradients $(300 \mathrm{mT} / \mathrm{m}$ maximum gradient strength, 21 $\mathrm{cm}$ inner bore size) was used for the study. A $2 \mathrm{~cm}$ diameter ${ }^{1} \mathrm{H}$ surface coil was used for signal reception in combination with a $15 \mathrm{~cm}$ inner diameter quadrature ${ }^{1} \mathrm{H}$ volume resonator for signal transmission. The center of the surface coil was carefully placed close to the tendon-bone junction by a trained physicist with previous experience in musculoskeletal MRI to ensure consistency in image acquisition. All specimens were aligned with their main axis along the main magnetic field $\left(\mathrm{B}_{0}\right)$ to minimize bias between the specimens due to the magic angle effect (30). A temperature of $4{ }^{\circ} \mathrm{C}$ was maintained for the duration of the MR imaging using a cooling system. For T2 mapping, a sagittal multi slice 2D multi-echo spin-echo (MESE) CPMG sequence with fat saturation was applied with the following parameters TR $=3.5 \mathrm{~s}$, TEs of the acquired echoes $=13.6,20.4,27.2$. 34.0, 40.8, 47.6, 54.4, $61.2 \mathrm{~ms}$ (the first echo at TE $=6.8 \mathrm{~ms}$ was not acquired), $\mathrm{FOV}=35 \times 26.3 \mathrm{~mm}^{2}$, matrix $=256 \times 192$, in-plane resolution $=0.137 \times 0.137 \mathrm{~mm}^{2}$, number of slices $=36$, slice thickness $=0.5 \mathrm{~mm}$, receiver bandwidth $(\mathrm{BW})=83.33 \mathrm{kHz}$, number of averages $=5$, total scan time $=1 \mathrm{~h} 52 \mathrm{~min}$. $\mathrm{T}^{*}$ mapping was performed using a 3D UTE sequence with twelve different TEs $=0.09$, $0.15,0.2,0.3,0.5,0.8,1.0,1.5,2.0,4.0,6.0,10.0 \mathrm{~ms}$. The sequence used a radial k-space acquisition scheme and per excitation one free induction decay was acquired. Other parameters for the sequence were: $\mathrm{TR}=12 \mathrm{~ms}$, flip angle $=8^{\circ}$, field of view $=45 \times 36 \times 26 \mathrm{~mm}^{3}$, number of spokes $=48,026, \mathrm{BW}=250 \mathrm{kHz}$, spatial resolution $=0.2 \times 0.2 \times 0.5$ $\mathrm{mm}^{3}$, total acquisition time for $\mathrm{T}^{*}$ imaging $=1 \mathrm{~h} 55 \mathrm{~min}$.

\section{Image and data analysis}

$\mathrm{T} 2$ and $\mathrm{T} 2{ }^{*}$ maps were generated by fitting the signal from the T2- and T2*-weighted datasets voxel wise to a monoexponential decay function using the Levenberg-Marquardt algorithm in custom programs written in MATLAB (MathWorks Inc., Natick, MA, USA) (31). The datasets and relaxation time maps were then interpolated to a nominal in-plane resolution of $47 \mu \mathrm{m} \times 47 \mu \mathrm{m}$ to match the different resolutions of the two imaging sequences and to improve visualization of the regions of interest (ROI) placement.

All MR images were analyzed by a blinded radiologist with previous experience in rabbit shoulder MRI. MRI slices in the middle portion of the SSP were selected. For $\mathrm{T} 2$ and $\mathrm{T} 2 *$ relaxation time analyses, three ROIs labelled 1 , 2 and 3 from distal to proximal were manually drawn on the first T2-weighted dataset to cover the complete thickness of the tendon from the bursal to the articular side. Each ROI covered approximately $0.6 \mathrm{~mm}$ of tendon length. For each tendon, the length covered by the 3 adjacent ROIs was approximately $2 \mathrm{~mm}$. To exclude the enthesis, the distal tendon ROI started $0.6 \mathrm{~mm}$ proximal to the greater tuberosity. Masks were generated from the ROIs and applied to the $\mathrm{T} 2$ and $\mathrm{T} 2^{*}$ maps to obtain the quantitative $\mathrm{T} 2$ and $\mathrm{T} 2 *$ relaxation times.

To ensure consistency between specimens, only ROIs containing 500-750 voxels were considered for analysis. Voxels which had suffered fit failure were excluded. Furthermore, any ROI where $>10 \%$ of voxels had suffered fit failure was not further considered for analysis. Stated another way, the number of "data generating" (successfully fit) voxels in an ROI had to be at least 450 , and the ratio of data-generating voxels to total voxels had to be at least 0.9 . ROIs were used to calculate $\mathrm{T} 2$ and $\mathrm{T} 2$ * average and standard deviation for individual ROIs and for the 3 combined ROIs from the same tendon.

Signal-to-noise ratio (SNR) of the two acquisitions were measured in 5 different tendons on the T2-weighted image (at TE $=13.6 \mathrm{~ms}$ ) and on the $\mathrm{T} 2{ }^{*}$-weighted image (at TE $=0.09 \mathrm{~ms}$ ) by taking the mean pixel intensity of the tendon and dividing it by the standard deviation of noise from a $\mathrm{ROI}$ in the background air.

\section{Statistical analysis}

The supraspinatus tendons were analyzed separately by two raters; one analyzing images of operated shoulders and the other the contralateral shoulders from the same rabbit. To measure inter-rater variability, the intra-class reliability assessment was used for 12 randomly selected rabbit shoulders. In the first analysis, average $( \pm$ STD) $\mathrm{T} 2$ times from individual ROIs in the same tendon and from the 7 groups (4 channeling and 3 no channeling) were compared using ANOVA with repeated measures. In the second analysis, the effect of channeling was tested by comparing the channeling and no-channeling groups mean $\mathrm{T} 2$ times from the 3 ROIs using an independent $t$-test. In the third analysis, the effect of surgical repair was tested by comparing mean T2 times between repaired and contralateral tendons at each time points using paired $t$-tests. In the fourth analysis, one-way ANOVA tested the effect of postoperative duration on $\mathrm{T} 2$ times for the experimental and contralateral groups independently. The 0 -week postoperative duration acted as a comparator for all subsequent time points. The strategy for data analysis 

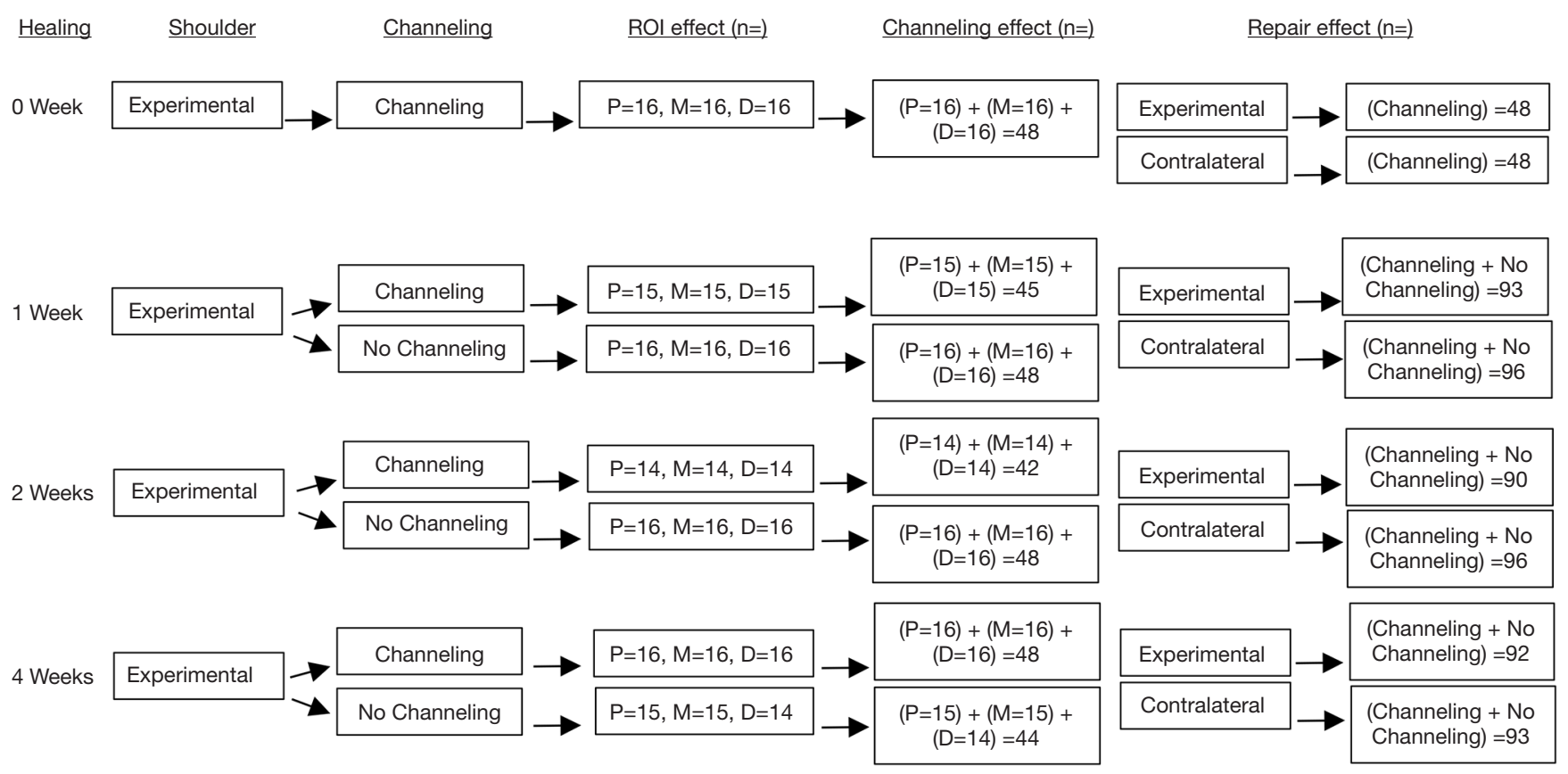

Figure 2 Strategy for data analysis and sample sizes. The number of regions of interest (ROI) analyzed are indicated for each group. P: proximal; M: middle; D: distal.

and sample sizes in each group is illustrated in Figure 2. $\mathrm{P}$ values less than 0.05 were considered statistically significant. Statistical analysis was performed using SPSS version 24.0 (IBM Corp., Armonk, NY, USA) and Microsoft Excel 2010 (Microsoft, Redmond, WA, USA).

\section{Results}

\section{Inter-rater agreement}

For the T2 sequence the SNR measurement of the tendon tissue resulted in $30.6 \pm 8.8$ (at $\mathrm{TE}=13.6 \mathrm{~ms}$ ), for the $\mathrm{T} 2$ * (UTE) sequence in $101.5 \pm 18.7$ (at TE $=0.09 \mathrm{~ms}$ ). T2 and $\mathrm{T} 2{ }^{*}$ map measures of experimental and of contralateral tendons were consistent between 2 raters with an inter-class coefficient correlation of 0.94 .

\section{T2 and T2*-weighted maps analysis}

T2 maps contained more voxels than $\mathrm{T}^{*}$ maps, resulting in more data-generating voxels (Figure $3 A, B$ and Tables 1,2). T2 maps from all repaired SSP tendons and most contralateral tendons met the pre-determined thresholds of 450 data-generating voxels (Table 1) and ratios of datagenerating voxels over total number of voxels of 0.9 (Table 2).
T2* maps of only half of the repaired and contralateral SSP tendons met the threshold of 0.9 and they were not further analyzed (Tables 1,2). An example of the $\mathrm{T} 2$ relaxation measurements and the mono-exponential fit from a single pixel within a contralateral tendon using $\mathrm{SI}(\mathrm{TE})=\mathrm{S} 0 \cdot \mathrm{e}$ (TE/T2) as fit model ( $\mathrm{SI}=$ signal intensity, $\mathrm{TE}=$ echo time, $\mathrm{S} 0=$ signal intensity at $\mathrm{TE}=0, \mathrm{~T} 2=\mathrm{T} 2$ relaxation time) is shown in Figure 4. T2 relaxation time from the fit resulted in $25.9 \mathrm{~ms}$ [ $95 \%$ confidence intervals: $(24.4 \mathrm{~ms} ; 29.1 \mathrm{~ms})$ ].

\section{Effect of ROI position on $T 2$ relaxation times}

T2 relaxation times of the distal, middle and proximal SSP tendon ROIs were not significantly different for both controls and operated shoulders (all $\mathrm{P}>0.05$ ) (Figure 5). The homogeneous distal-to-proximal $\mathrm{T} 2$ times were observed in the repaired and contralateral SSP tendons, at the 4 postoperative durations and whether or not there was bone channeling (Figure 5). Given the lack of distal-toproximal gradient, T2 data from the 3 ROIs were averaged to further compare groups.

\section{Effect of channeling on $T 2$ relaxation time}

Bone channeling had no significant effects on T2 relaxation 
A

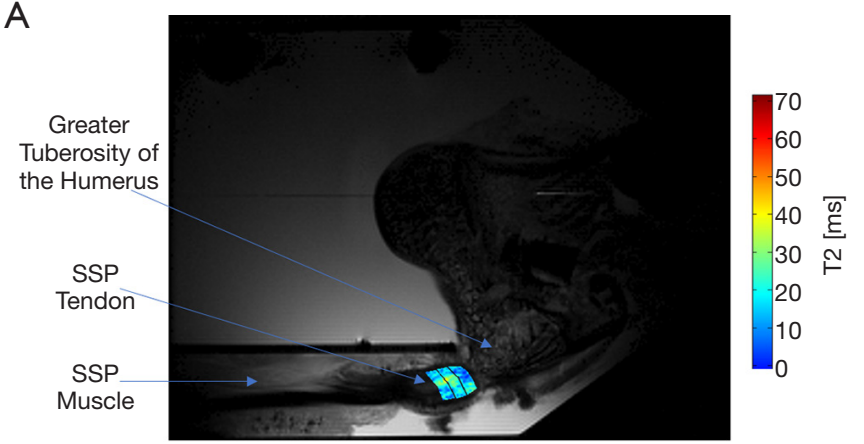

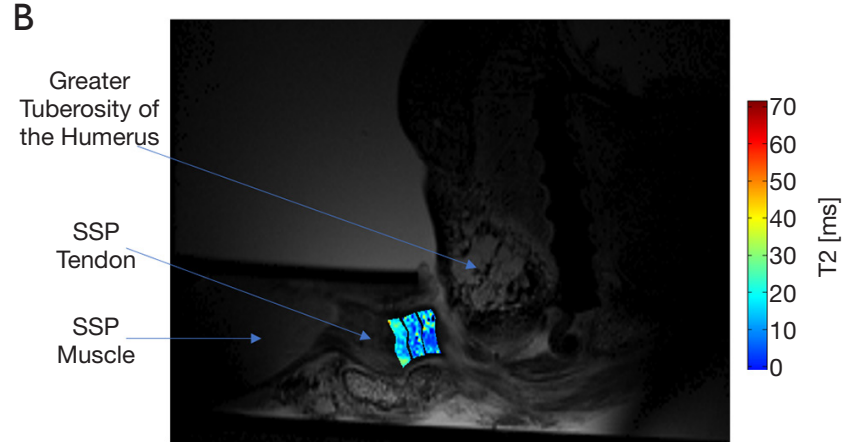

Figure 3 Representative T2 maps of the repaired supraspinatus (SSP) tendons 1 week after detachment and no healing time (Figure 3 A) and after 4 postoperative weeks (Figure $3 B$ ) are represented as color-coded region of interest (ROI) overlays on the anatomical images. Colors indicate voxels generating a signal that reached the threshold of 450 in the ROI shown in color proportional to the relaxation time and according to scale.

Table 1 Mean number of voxel and data-generating voxels—combined regions of interest (ROI)

\begin{tabular}{|c|c|c|c|c|}
\hline Relaxation type & Postoperative duration & Intervention & \multicolumn{2}{|c|}{ All ROls } \\
\hline \multirow[t]{3}{*}{ T2 } & Week 0 & Contralateral & $695.5 \pm 188.2$ & $675.3 \pm 190.1$ \\
\hline & & Experimental & $781.8 \pm 89.3$ & $751.2 \pm 100.1$ \\
\hline & Week 4 & Contralateral & $675.1 \pm 115.9$ & $671.4 \pm 112.1$ \\
\hline \multirow[t]{4}{*}{$\mathrm{T}^{*}$} & Week 0 & Contralateral & $542.8 \pm 153.9$ & $426.2 \pm 190.3$ \\
\hline & & Experimental & $564.9 \pm 128.6$ & $414.2 \pm 180.6$ \\
\hline & Week 4 & Contralateral & $613.3 \pm 165.4$ & $456.9 \pm 184.6$ \\
\hline & & Experimental & $583.3 \pm 120.4$ & $389.8 \pm 199.6$ \\
\hline
\end{tabular}

ROIs were manually drawn on the first T2-weighted dataset from experimental and contralateral groups at 0 and 4 weeks. The average number of voxels $( \pm$ STD) for all ROls with a minimum of 450 voxels is indicated for each group. Data-generating voxels corresponded to fitted voxels and ROls with a minimum of $90 \%$ of fitted voxels were considered for analysis. The ratio of data-generating voxels to total voxels was set at a minimum of 0.9 .

time at all postoperative durations (all $\mathrm{P}>0.05$ ) (Figure 6). Given the lack of effect of bone channeling, T2 data from the channeling and no-channeling groups were averaged to further compare groups.

\section{Effect of SSP tendon tear on $T 2$ relaxation time}

T2 of SSP tendon proper that were repaired 1 week after tendon tear was significantly lower compared to the contralateral SSP $(20.0 \pm 3.4 \mathrm{~ms} v s .25 .6 \pm 3.9 \mathrm{~ms} ; \mathrm{P}<0.05$; Figure 7).

\section{Effect of surgical repair for up to 4 postoperative weeks on $T 2$ relaxation time}

T2 increased with postoperative duration to $22.7 \pm 3.1 \mathrm{~ms}$; $23.3 \pm 3.9 \mathrm{~ms}$ and to $24.0 \pm 5.1 \mathrm{~ms}$ respectively after 1,2 and 4 postoperative weeks; all were significantly higher than the operative day (week 0; 20.0 $\pm 3.4 \mathrm{~ms}$; all $\mathrm{P}<0.05$; Figure 7). No such increase in $\mathrm{T} 2$ relaxation time with postoperative duration was detected in contralateral SSP (Figure 7). Despite the postoperative time increase, repaired SSP tendons T2 times remained statistically significantly lower 
Table 2 Ratio of data-generating voxels/number of voxels—combined regions of interest (ROI)

\begin{tabular}{lllc}
\hline \multirow{2}{*}{ Relaxation type } & Postoperative duration & Intervention & Data-generating voxels/number of voxels \\
\cline { 3 - 4 } & Week 0 & Contralateral & 0.971 \\
\hline T2 Week 4 & & Experimental & 0.961 \\
& Week 0 & Contralateral & 0.994 \\
T2* & & Experimental & 0.993 \\
& & Contralateral & 0.785 \\
& Week 4 & Experimental & 0.734 \\
& & Contralateral & 0.749 \\
\hline
\end{tabular}

The ratio of data-generating voxels to total voxels for experimental and contralateral groups at weeks 0 and 4 and was set at a minimum of 0.9 .

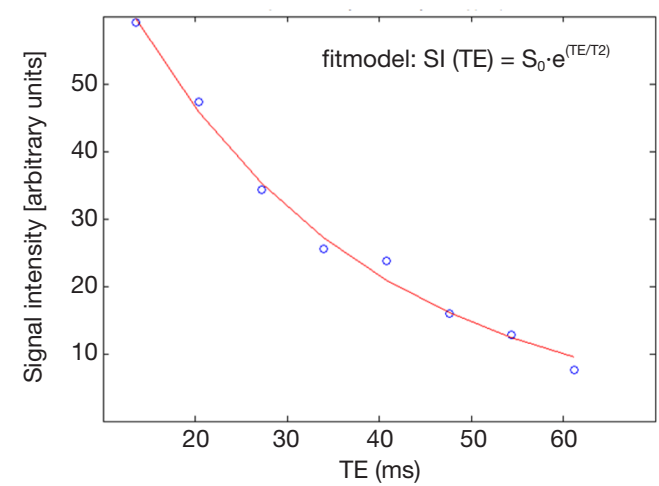

Figure 4 Example of the T2 relaxation measurements (circles) and the mono- exponential fit (line) from a single pixel within the rabbit supraspinatus tendon using $\mathrm{SI}(\mathrm{TE})=\mathrm{S} 0 \cdot \mathrm{e}(\mathrm{TE} / \mathrm{T} 2)$ as fit model (SI = signal intensity, $\mathrm{TE}=$ echo time, $\mathrm{S} 0=$ signal intensity at $\mathrm{TE}=0$, $\mathrm{T} 2=\mathrm{T} 2$ relaxation time). $\mathrm{T} 2$ from the fit resulted in $25.9 \mathrm{~ms}[95 \%$ confidence intervals: $(24.4 \mathrm{~ms} ; 29.1 \mathrm{~ms})]$.

than their contralateral tendons even after 4 postoperative weeks (all $\mathrm{P}<0.05$; Figure 7).

\section{Discussion}

We found significantly shorter T2 relaxation times in SSP tendon proper that improved with time after surgical repair but had not reached contralateral values after 4 postoperative weeks. By evaluating the effects of channeling ahead of repair surgery on SSP tendon compositional healing, this study has provided some indication of the ineffectiveness of this biological augmentation strategy.

We report reliable and reproducible $\mathrm{T} 2$ relaxation times measured serially 1 week after SSP tendon detachment and up to 4 weeks after surgical repair with a lateral anchor. Bi-exponential relaxation time fitting requires generally high SNR, a high ratio of the two relaxation time components, and typically a large number of echoes to minimize the error in the fitting process (32). Although the UTE T2* sequence SNR in our study was relatively high, we performed a mono-exponential relaxation decay analysis due to the robustness of the mono-exponential fitting approach. The tendon SNR of the T2 sequence was sufficient to perform a classical single component T2 decay fit (33). Therefore, we applied also a mono-exponential decay analysis for $\mathrm{T} 2$ to achieve robust fitting results. The mono-exponential analysis of $\mathrm{T} 2$ in this study represents a weighted mean value from different transversal relaxation time components. The shorter components originated from bound water while the longer components originated from free water (34). Tendons contain $\sim 70 \%$ water partly bound with collagens mainly type I and proteoglycans $(14,35)$. In a T2 mapping study of asymptomatic volunteers at 3T, mean T2 values reported were $33.6 \pm 5.3 \mathrm{~ms}$ (36). Patients with partial tears and minimally retracted full-thickness tears had higher mean T2 values: $43.9 \pm 12.7 \mathrm{~ms}(36)$. Our T2 values of contralateral rabbit SSP tendon proper (around $25 \mathrm{~ms}$ ) were shorter, supporting that changes in tendon structure after surgical repair may be detectable by MRI.

We found shorter T2 relaxation times in detached 

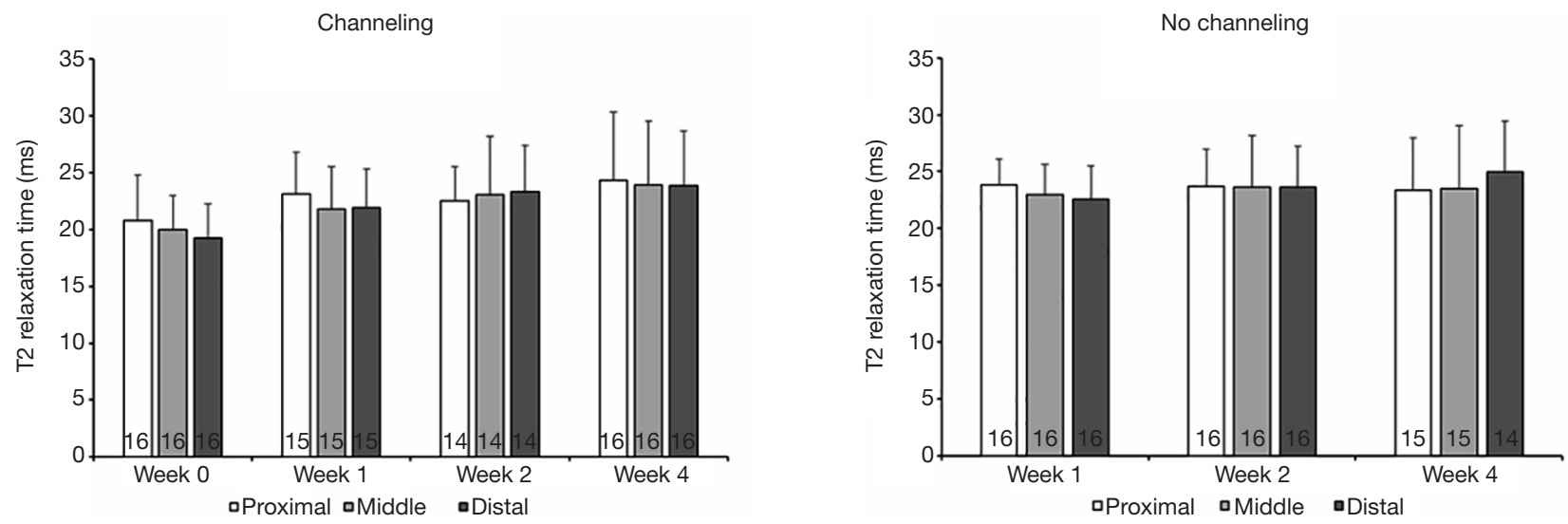

Figure 5 T2 relaxation times (ms) in the proximal (white), middle (grey) and distal (black) regions of rabbit supraspinatus (SSP) tendons 1 week after detachment with channeling or no-channeling as well as contralateral after $0,1,2$, and 4 weeks of healing. Relaxation times expressed as the mean $( \pm$ STD) and number of shoulders indicated at bottom of each bar. The effect of region of interest (ROI) location was measured by comparing T2 relaxation times for the 3 tendon regions from the same time point and same intervention; channeling or no channeling. All $\mathrm{P}>0.05$.

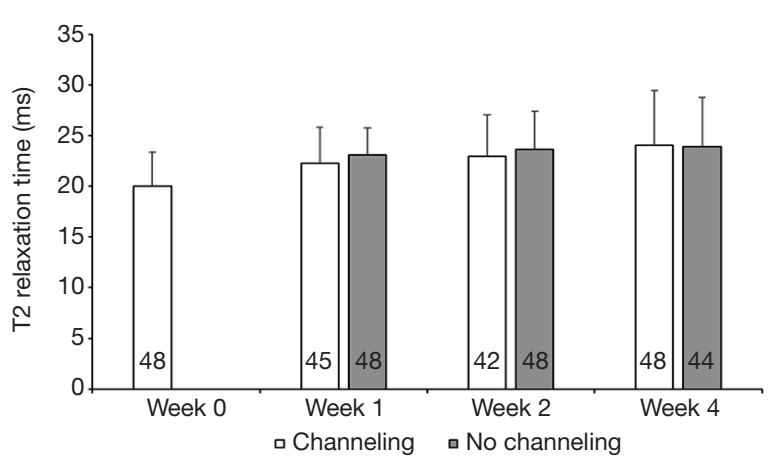

Figure 6 Averaged T2 relaxation time (ms) for the three tendon regions of interest (ROIs) with channeling (white) or without (grey) after $0,1,2$, and 4 weeks of healing. Relaxation time expressed as the mean \pm STD of the three ROIs (given there was no significant differences between ROIs: Figure 5) and number of shoulders indicated on each bar. The effect of channeling was measured by comparing channeling to no-channeling for the same healing time point using unpaired $t$-test. All $\mathrm{P}$ values $>0.05$. The 0 weeks of healing time point only had the channeling group. Sample size per group indicated on the bars.

SSP tendons, confirming our first hypothesis. These are consistent with tendon disuse where detached and retracted tendons are no longer transmitting any load. In this study,

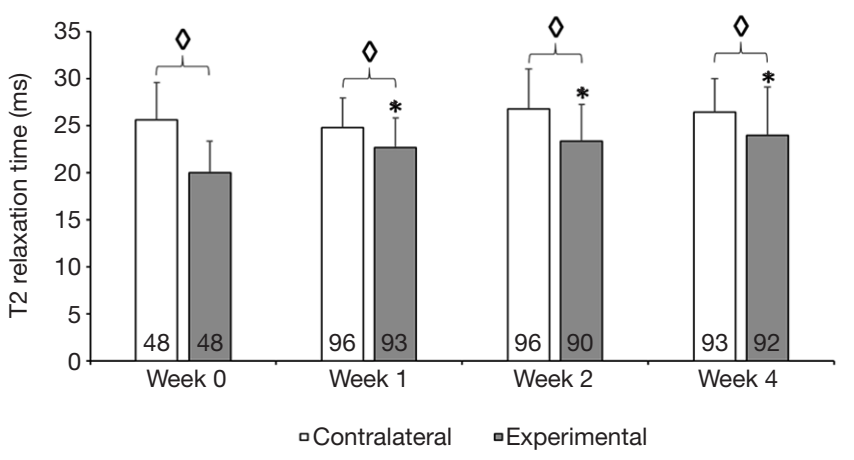

Figure 7 Averaged T2 relaxation times (ms) for repaired supraspinatus (SSP) tendons 1 week after detachment and 0,1 , 2 , and 4 weeks of healing (grey) and for contralateral (white). Relaxation times expressed as the mean $( \pm$ STD) of channeling and of no channeling specimens (given there was no significant differences between channeling and no-channeling: Figure 6) and number of shoulders indicated on each bar. The effect of repair over time was measured by comparing values for the experimental and contralateral shoulders at individual time points $(\diamond: \mathrm{P}<0.05)$. Healing time of 1,2 , and 4 weeks, significantly increased T2 relaxation time compared to week $0\left(^{*}\right.$ : $\left.\mathrm{P}<0.05\right)$. Time had no effect on contralateral (all $\mathrm{P}>0.05)$.

the duration of SSP tear was 1 week. This is important clinically as poor tendon proper quality can complicate the repair with poor suture purchase. We found that surgical 
repair partially overturned the $\mathrm{T} 2$ changes progressively over 4 postoperative weeks which confirmed our second hypothesis. These reveal a progressive return towards normal of the SSP tendon proper structural properties and potential benefits of the repair surgery that can be monitored by imaging. The imaging also reliably correlated with the mechanical restoration of SSP tendon repairs on the same samples (37). However, the recovery was incomplete after 4 postoperative weeks. This is important clinically since re-ruptures were described to happen more frequently at the tendon proper rather than at the repair enthesis site (16). Therefore, low postoperative $\mathrm{T} 2$ indicating incomplete biochemical and mechanical healing could constitute clinically important indication of incomplete healing and considered in postoperative rehabilitation protocols. It is important to differentiate the changes at the tendon proper from the changes at the SSP tendon enthesis. The 4-zone enthesis is composed of mineralized and unmineralized fibrocartilage. Similarly, the different tissues at the tendon proper and at the enthesis responded differently to tearing and surgical repair (27). Postoperatively, increased T2 were measured at the repair site where degenerated distal end, inflammation and postsurgical reaction at the SSP enthesis are paramount, constituting an entirely different process from what we identified at the SSP tendon proper. Increased T1 and T2 contrast were associated with tendinitis (38).

The current study represents an important step towards the transition to quantitative imaging of the SSP tendon and provides preliminary data for clinical translation to patient outcome and surgical studies. Quantitative parameters of knee MR imaging at 3T and at 7T with similar sequences and scan times were compared to evaluate relevant anatomical areas including tendons (39). Imaging accelerations such as parallel imaging and compressed sensing can also be applied (40). Ultra-high-field MRI at $7 \mathrm{~T}$ sequences with higher voxel-volume-adjusted SNR compared to $3 \mathrm{~T}$ can potentially improve diagnostic accuracy.

Interventions aimed at augmenting rotator cuff repairs such as bone channeling, as assessed in the current study, are investigated for their potential benefits. The lack of effect of bone channeling on $\mathrm{T} 2$ relaxation times is consistent with previous data recorded at the enthesis (27) and with mechanical data from the same specimens (37) and supported our third hypothesis. Reasons for the lack of efficacy may be size of channel, closing of channel in the preoperative period, or lack of contact between channel and tendon postoperatively.

\section{Limitations}

The rabbit SSP tendon detachment model may not accurately reflect the human SSP tendon tear. We performed ex vivo imaging and postmortem cell degeneration that could have modified the extracellular matrix and thus altered the tendon biochemical properties. This limitation would equally affect all specimens, experimental and controls, and was minimized by applying deep-freezing at $-40{ }^{\circ} \mathrm{C}$ immediately after harvest and by thawing and imaging at $4^{\circ} \mathrm{C}$. Repair sutures going through the SSP tendon could theoretically have decreased T2 time. The use of rabbits of similar age instead of contralateral shoulders could have eliminated the possible influence of a training effect. The stability of T2 signal in 112 different tendons throughout the duration of the experiment argues against a training effect and supports contralateral SSP tendons as valid controls in this study. The lack of histologic and morphologic assessments to correlate with imaging results also represents a limitation.

\section{Conclusions}

SSP tendon tear of 1-week duration decreased T2 relaxation time as measured with $7 \mathrm{~T}$ MRI. Surgical repair progressively restored $\mathrm{T} 2$ times without reaching contralateral levels after 4 postoperative weeks. Bone channeling did not accelerate SSP tendon recovery. Quantitative T2 maps using 7T MRI identified disuse of the SSP tendon proper after a SSP tear and the benefits of surgical repair which support its use for future preoperative and postoperative investigations of SSP tendons.

\section{Acknowledgments}

We acknowledge the assistance of the University of Ottawa Animal Care and Veterinary Services staff for surgical assistance, Dr. J. W. Pollock who helped developed the surgical technique, Ms. Y. Nie for specimen preparation, and Dr. Hans K. Uhthoff for his advice. Arthrex Inc. provided surgical instrumentation and anchors for the repair surgeries.

Funding: This work was supported by the Canadian Institutes of Health Research; contract grant number: FRN 
110995.

\section{Footnote}

Conflicts of Interest: All authors have completed the ICMJE uniform disclosure form (available at http://dx.doi. org/10.21037/qims-20-1343). Dr. Guy Trudel and Dr. Odette Laneuville report that they are the recipients of the research grant from the Canadian Institutes of Health Research. Dr. Peder E. Z. Larson receives research support from GE Healthcare and royalty payments for patents related to the work. Dr. Samuel Duchesne-Bélanger, Dr. Justin Thomas, Dr. Greg O. Cron, Dr. Mark Schweitzer, Dr. Adnan Sheikh, Dr. Hakim Louati MEng report that this work was supported by the Canadian Institutes of Health Research: grant number FRN 110995. Funds were used to purchase material, cost of services including MRI services, animal surgeries and care, and to cover the salaries of staff. The other author has no conflicts of interest to declare.

Ethical Statement: The authors are accountable for all aspects of the work in ensuring that questions related to the accuracy or integrity of any part of the work are appropriately investigated and resolved. Experiments were performed under a project license (protocol \#ME184) granted by the ethics board of University of Ottawa Animal Care Committee, in compliance with the national or institutional guidelines for the care and use of animals.

Open Access Statement: This is an Open Access article distributed in accordance with the Creative Commons Attribution-NonCommercial-NoDerivs 4.0 International License (CC BY-NC-ND 4.0), which permits the noncommercial replication and distribution of the article with the strict proviso that no changes or edits are made and the original work is properly cited (including links to both the formal publication through the relevant DOI and the license). See: https://creativecommons.org/licenses/by-nc-nd/4.0/.

\section{References}

1. McGarvey C, Harb Z, Smith C, Houghton R, Corbett S, Ajuied A. Diagnosis of rotator cuff tears using a 3-Tesla MRI versus 3-Tesla MRA: a systematic review and metaanalysis. Skeletal Radiol 2016;45:251-61.

2. McCrum E. MR Imaging of the Rotator Cuff. Magn Reson Imaging Clin N Am 2020;28:165-79.

3. Sher JS, Iannotti JP, Williams GR, Herzog RJ, Kneeland
JB, Lisser S, Patel N. The effect of shoulder magnetic resonance imaging on clinical decision making. J Shoulder Elbow Surg 1998;7:205-9.

4. Gladstone JN, Bishop JY, Lo IK, Flatow EL. Fatty infiltration and atrophy of the rotator cuff do not improve after rotator cuff repair and correlate with poor functional outcome. Am J Sports Med 2007;35:719-28.

5. Jain NB, Higgins LD, Losina E, Collins J, Blazar PE, Katz JN. Epidemiology of musculoskeletal upper extremity ambulatory surgery in the United States. BMC Musculoskelet Disord 2014;15:4-7.

6. Karjalainen TV, Jain NB, Heikkinen J, Johnston RV, Page CM, Buchbinder R. Surgery for rotator cuff tears. Cochrane Database Syst Rev 2019;12:CD013502.

7. Agout C, Berhouet J, Bouju Y, Godeneche A, Collin P, Kempf JF, Favard L. Clinical and anatomic results of rotator cuff repair at 10 years depend on tear type. Knee Surg Sports Traumatol Arthrosc 2018;26:2490-7.

8. Frangiamore S, Dornan GJ, Horan MP, Mannava S, Fritz EM, Hussain ZB, Moatshe G, Godin JA, Pogorzelski J, Millett PJ. Predictive Modeling to Determine Functional Outcomes After Arthroscopic Rotator Cuff Repair. Am J Sports Med 2020;48:1559-67.

9. Bydder GM. Review. The Agfa Mayneord lecture: MRI of short and ultrashort $\mathrm{T} 2$ and $\mathrm{T} 2{ }^{*}$ components of tissues, fluids and materials using clinical systems. Br J Radiol 2011;84:1067-82.

10. Bachmann E, Rosskopf A, Götschi T, Klarhofer M, Deligianni X, Hilbe M, Pfirrmann CWA, Snedeker JG, Fischer MA. T1- and T2*-Mapping for Assessment of Tendon Tissue Biophysical Properties. A Phantom MRI Study. Invest Radiol 2019;54:212-20.

11. Kijowski R, Thurlow P, Blankenbaker D, LiuF, McGuine T, Li G, Tuite M. Preoperative MRI Shoulder Findings Associated With Clinical Outcome 1 Year After Rotator Cuff Repair. Radiology 2019;291:722-9.

12. Riley GP, Harrall RL, Constant CR, Chard MD, Cawston TE, Hazleman BL. Tendon degeneration and chronic shoulder pain: changes in the collagen composition of the human rotator cuff tendons in rotator cuff tendinitis. Ann Rheum Dis 1994;53:359-66.

13. Järvinen M, Jozsa L, Kannus P, Järvinen TL, Kvist M, Leadbetter W. Histopathological findings in chronic tendon disorders. Scand J Med Sci Sports 1997;7:86-95.

14. DE Giorgi S, Saracino M, Castagna A. Degenerative disease in rotator cuff tears: what are the biochemical and histological changes? Joints 2014;2:26-8. 
15. Ryan CN, Sorushanova A, Lomas AJ, Mullen AM, Pandit A, Zeugolis DI. Glycosaminoglycans in tendon physiology, pathophysiology, and therapy. Bioconjug Chem 2015;26:1237-51.

16. Uhthoff HK, Trudel G, Himori K. Relevance of pathology and basic research to the surgeon treating rotator cuff disease. J Orthop Sci 2003;8:449-56.

17. Hinsley H, Nicholls A, Daines M, Wallace G, Arden N, Carr A. Classification of rotator cuff tendinopathy using high definition ultrasound. Muscles Ligaments Tendons J 2014;4:391-7.

18. Chang EY, Du J, Chung CB. UTE imaging in the musculoskeletal system. J Magn Reson Imaging 2015;41:870-83.

19. Gatehouse PD, Bydder GM. Magnetic resonance imaging of short T2 components in tissue. Clin Radiol 2003;58:1-19.

20. Ma YJ, West J, Nazaran A, Cheng X, Hoenecke H, Du J, Chang EY. Feasibility of using an inversion-recovery ultrashort echo time (UTE) sequence for quantification of glenoid bone loss. Skeletal Radiol 2018;47:973-80.

21. Anz AW, Lucas EP, Fitzcharles EK, Surowiec RK, Millett PJ, Ho CP. MRI T2 mapping of the asymptomatic supraspinatus tendon by age and imaging plane using clinically relevant subregions. Eur J Radiol 2014;83:801-5.

22. Juras V, Mlynarik V, Szomolanyi P, Valkovic L, Trattnig S. Magnetic Resonance Imaging of the Musculoskeletal System at 7T: Morphological Imaging and Beyond. Top Magn Reson Imaging 2019;28:125-35.

23. Krepkin K, Bruno M, Raya JG, Adler RS, Gyftopoulos S. Quantitative assessment of the supraspinatus tendon on MRI using T2/T2* mapping and shearwave ultrasound elastography: a pilot study. Skeletal Radiol 2017;46:191-9.

24. Trattnig S, Zbýň S, Schmitt B, Friedrich K, Juras V, Szomolanyi P, Bogner W. Advanced MR methods at ultrahigh field (7 Tesla) for clinical musculoskeletal applications. Eur Radiol 2012;22:2338-46.

25. Han M, Larson PE, Liu J, Krug R. Depiction of Achilles tendon microstructure in vivo using high-resolution 3-dimensional ultrashort echo-time magnetic resonance imaging at 7 T. Invest Radiol 2014;49:339-45.

26. Fukawa T, Yamaguchi S, Watanabe A, Sasho T, Akagi R, Muramatsu Y, Akatsu Y, Katsuragi J, Endo J, Osone F, Sato Y, Okubo T, Takahashi K. Quantitative assessment of tendon healing by using MR T2 mapping in a rabbit Achilles tendon transection model treated with platelet- rich plasma. Radiology 2015;276:748-55.

27. Trudel G, Melkus G, Cron GO, Louati H, Sheikh A, Larson PEZ, Schweitzer M, Lapner P, Uhthoff HK, Laneuville O. Imaging of the rabbit supraspinatus enthesis at 7 Tesla: a 4-week time course after repair surgery and effect of channeling. J Magn Reson Imaging 2017;46:461-7.

28. Uhthoff HK, Coletta E, Trudel G. Effect of timing of surgical SSP tendon repair on muscle alterations. J Orthop Res 2014;32:1430-5.

29. Boileau P, Brassart N, Watkinson DJ, Charles M, Hatzidakis AM, Krishnan SG. Arthroscopic repair of fullthickness tears of the supraspinatus: does the tendon really heal? J Bone Joint Surg Am 2005;87:1229-40.

30. Milford D, Rosbach N, Bendszus M, Heiland S. MonoExponential Fitting in T2-Relaxometry: Relevance of Offset and First Echo. PLoS One 2015;10:e0145255.

31. Majumdar S, Gmitro A, Orphanoudakis SC, Reddy D, Gore JC. An estimation and correction scheme for system imperfections in multiple-echo magnetic resonance imaging. Magn Reson Med 1987;4:203-20.

32. Anastasiou A, Hall LD. Optimisation of T2 and M0 measurements of bi-exponential systems. Magn Reson Imaging 2004;22:67-80.

33. Raya JG, Dietrich O, Horng A, Weber J, Reiser MF, Glaser C. T2 measurement in articular cartilage: impact of the fitting method on accuracy and precision at low SNR. Magn Reson Med 2010;63:181-93.

34. Peto S, Gillis P, Henri VP. Structure and dynamics of water in tendon from NMR relaxation measurements. Biophys J 1990;57:71-84.

35. Juras V, Apprich S, Szomolanyi P, Bieri O, Deligianni $\mathrm{X}$, Trattnig S. Bi-exponential T2 analysis of healthy and diseased Achilles tendons: an in vivo preliminary magnetic resonance study and correlation with clinical score. Eur Radiol 2013;23:2814-22.

36. Ganal E, Ho CP, Wilson KJ, Surowiec R, Smith WS, Dornan GJ, Millett PJ. Quantitative MRI characterization of arthroscopically verified supraspinatus pathology: comparison of tendon tears, tendinosis and asymptomatic supraspinatus tendons with T2 mapping. Knee Surg Sports Traumatol Arthrosc 2016;24:2216-24.

37. Louati H, Uhthoff HK, Culliton K, Laneuville O, Lapner P, Trudel G. Supraspinatus tendon repair using anchors: a biomechanical evaluation in the rabbit. J Orthop Surg Res 2018;13:64-70.

38. Lee YH, Kim S, Lim D, Song HT, Suh JS. MR quantification of the fatty fraction from $\mathrm{T} 2 *$ 
corrected Dixon fat/water separation VolumeInterpolated Breath-hold Examination (VIBE) in the assessment of muscle atrophy in rotator cuff tears. Acad Radiol 2015;22:909-17.

39. Camarda L, Grassedonio E, Albano D, Galia M, Midiri M, D'Arienzo M. MRI evaluation to predict tendon size for

Cite this article as: Trudel G, Duchesne-Bélanger S, Thomas J, Melkus G, Cron GO, Larson PEZ, Schweitzer M, Sheikh A, Louati H, Laneuville O. Quantitative analysis of repaired rabbit supraspinatus tendons ( \pm channeling) using magnetic resonance imaging at 7 Tesla. Quant Imaging Med Surg 2021;11(8):3460-3471. doi:10.21037/qims-20-134 knee ligament reconstruction. Muscles Ligaments Tendons J 2018;7:478-84.

40. Boucneau T, Cao P, Tang S, Han M, Xu D, Henry RG, Larson PEZ. In vivo characterization of brain ultrashort-T2 components. Magn Reson Med 2018;80:726-35. 